
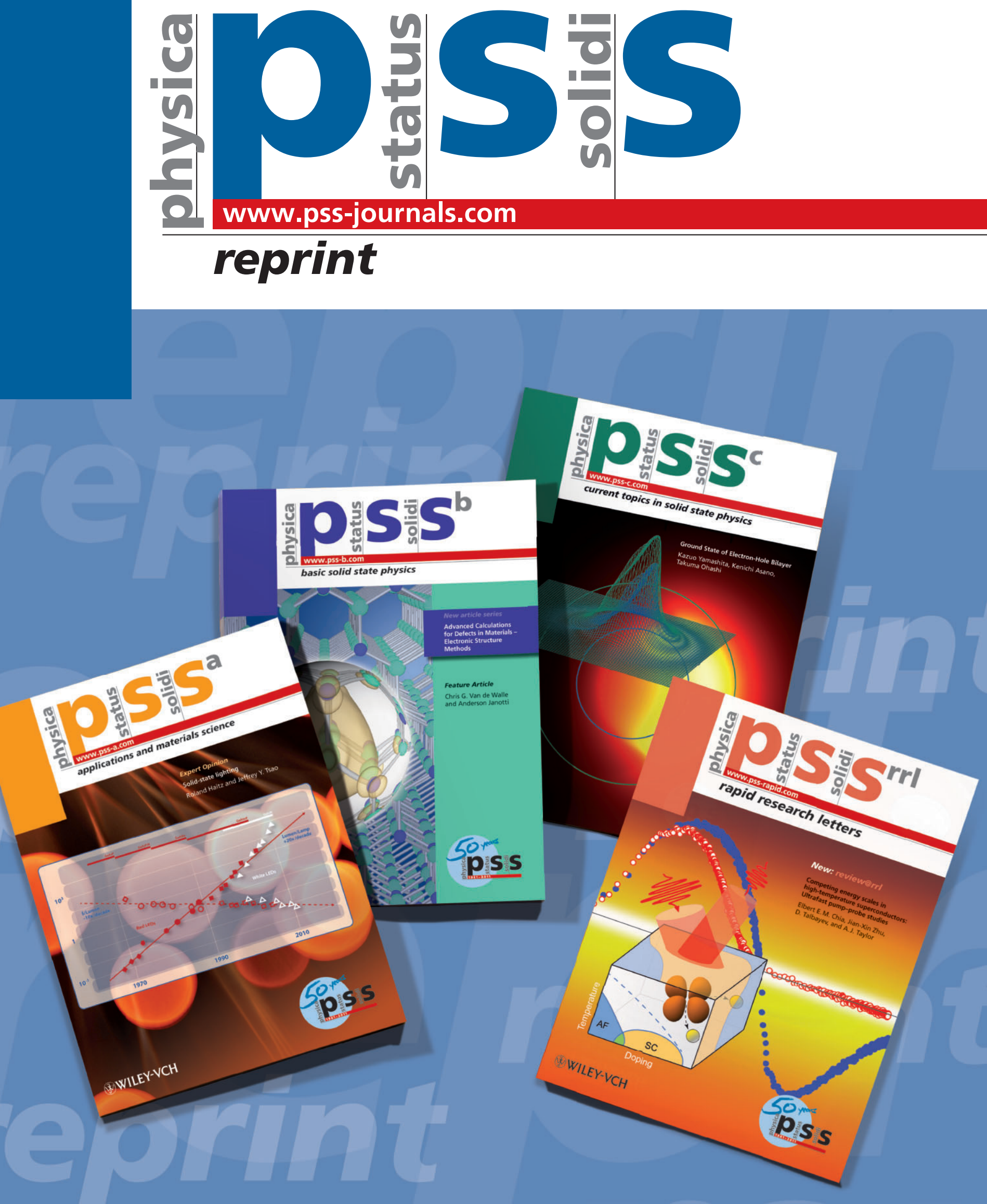


\title{
Effect of the pn junction engineering on Si microwire-array solar cells
}

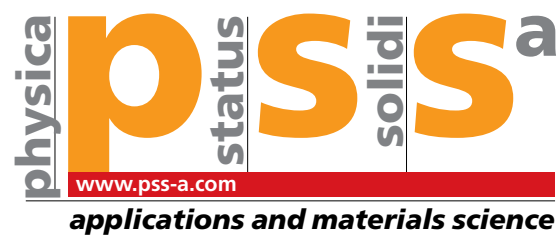

\author{
A. Dalmau Mallorquí, F. M. Epple, D. Fan, O. Demichel, and A. Fontcuberta i Morral* \\ Laboratoire des Matériaux Semiconducteurs, École Polytechnique Fédérale de Lausanne, 1015 Lausanne, Switzerland
}

Received 12 March 2012, revised 18 April 2012, accepted 19 April 2012

Published online 18 May 2012

Keywords depletion width, diffusion profile, microwire solar cells, $p-n$-junction, recombination

*Corresponding author: e-mail anna.fontcuberta-morral@epfl.ch, Phone: +41-21-6937394, Fax: +41-21-6934755

We report on the impact of the doping concentration design on the performance of silicon microwire arrays as photovoltaic devices. We have fabricated arrays with different $p$ - and $n$ doping profiles and thicknesses, obtaining mean efficiencies as high as $9.7 \%$ under AM $1.5 \mathrm{G}$ solar illumination. The results reveal the importance of scaling the microwire diameter with the depletion width resulting from doping concentrations. The doping of the core should be kept low in order to reduce bulk recombination. Furthermore, the thickness of the $n$-shell should be kept as thin as possible to limit the emitter losses.
1 Introduction Photovoltaic production and research has grown exponentially over the last twenty years due to the rising economic and environmental cost of fossil fuels. However, the cost per unit of energy produced remains high compared to typical grid prices. Thus, new concepts and technologies are arising to increase the efficiency/cost ratio [1]. Within this framework, nanowire-based $p-n$ radial junctions constitute a promising approach. By enhancing light trapping over a wide range of wavelengths and decoupling the direction of light absorption and carrier collection, these architectures can improve the efficiency of photovoltaic devices and ease the quality requirements of the materials of which they are composed [2,3]. Recent studies in $\mathrm{Si}$ nanowires have reported efficiencies up to $9 \%$ for single-wire devices [4] and $11 \%$ for an array of vertical wires [5]. Nanowire-based devices offer scope for further improvement by increasing light absorption (either by changing the wire geometry and the array configuration [6-9] or adding scattering nanoparticles between the wires [10]) or by decreasing the surface recombination via surface passivation $[5,11]$.

In the present work, we study how the particular design of a radial $p n$ junction affects the light conversion efficiency of the device. For this, we vary the microwire diameter, doping concentrations and junction depth. The results are evaluated by measuring the conversion efficiency under AM 1.5 conditions and the external quantum efficiency (EQE).
2 Experimental details A scheme of the solar cell fabrication process is shown in Fig. 1. Ordered arrays of microwires are fabricated by a combination of optical lithography and deep reactive ion etching on a $380 \mu \mathrm{m}$ thick Czochralski $\langle 100\rangle p$-doped Si wafer [12]. Hexagonally packed $45-\mu \mathrm{m}$ long wires with diameters of $1.86,2.4$, and $3.1 \mu \mathrm{m}$, spaced 7,8 , and $10 \mu \mathrm{m}$, respectively, are obtained. Three substrates with different resistivities are used: $0.1-0.5,1-5$, and $1-10 \Omega \mathrm{cm}$. The $n$-shell is formed by diffusing $\mathrm{POCl}_{3}$ for $15 \mathrm{~min}$ at 850 and $900{ }^{\circ} \mathrm{C}$. In order to diminish the defect concentration at the surface of the wires caused by the etching process, a thermal oxidation step is added right after the diffusion. The oxide layer is stripped off by wet etching in HF. A conformal indium tin oxide (ITO) layer with a nominal thickness of $70 \mathrm{~nm}$ is sputtered on the front side as an $n$-contact and $200 \mathrm{~nm}$ of $\mathrm{Al}$ as a rear contact. Optical and electrical properties of ITO were measured with an ellipsometer (Sopra GES-5E) and a resistivity meter four-point measurements, respectively, obtaining a transmittance in the visible range of $96 \%$ and a resistivity of $0.2-0.4 \mathrm{~m} \Omega \mathrm{cm}$, which proves ITO as a suitable front contact. In order to get a better contact for electrical measurements, four Ti/Au pads $(10 / 200 \mathrm{~nm})$ are evaporated around the array. The surface of the arrays is $5.4 \times 5.4 \mathrm{~mm}^{2}$. A scanning electron microscopy (SEM) image of a typical array is shown in Fig. 1f. Wires are uniform in diameter along the whole length and across de wafer. 
(a)

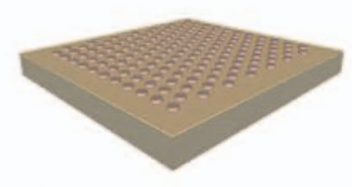

(e)

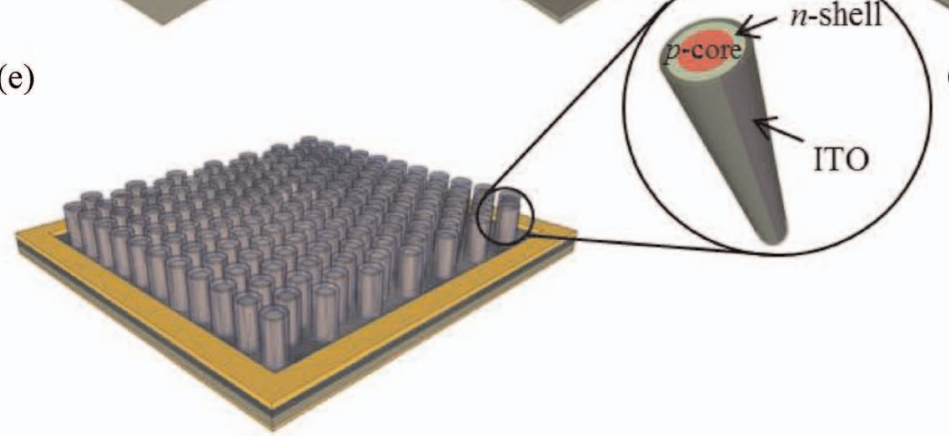

(c)

(b)

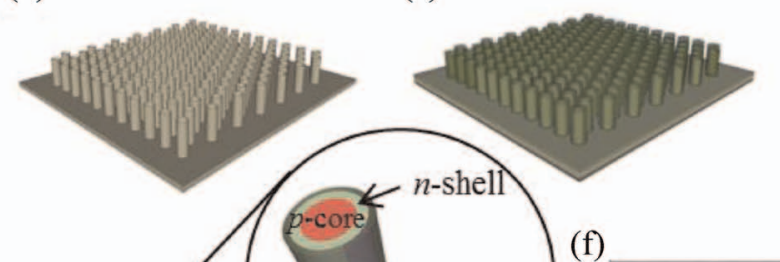

(d)

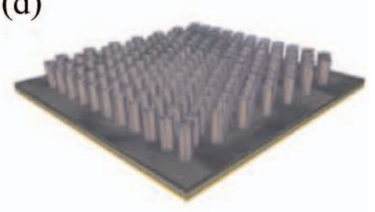

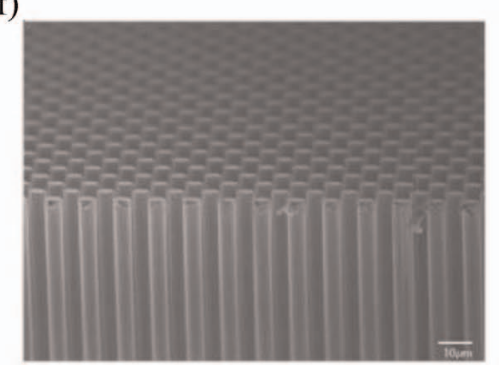

Figure 1 (online color at: www.pss-a.com) Fabrication schematic of radial $p-n$ junction Si microwire arrays: (a) patterning of a $p$-type $\mathrm{Si}$ wafer by means of photolithography; (b) deep reactive ion etching to obtain $p$-core microwire arrays; (c) $\mathrm{POCl}_{3}$ diffusion to create an $n$-shell; (d) ITO and Al sputtering as front and rear contacts, respectively; and (e) evaporation of Ti/Au contacts around the arrays. A detail of the resulting cross-section is also depicted. (f) SEM image of a fabricated microwire array viewed at $\sim 30^{\circ}$ tilt.

3 Results and discussion The doping concentration profiles of the junctions were measured by capacitancevoltage measurements (Wafer Profiler CVP21) on flat substrates exposed to the same diffusion treatments. The results transposed to the wire $p n$ junctions are shown in Fig. 2 (the erratic profile of the $p$-doped region is due to an experimental aberration resulting from the sharpness of the junction [13]). As expected from the fabrication process, the doping concentration of the core is constant, while the doping concentration at the shell decreases from the surface to the core following the diffusion profile depicted by Fick's law [14]:

$$
\frac{\partial N_{\mathrm{D}}}{\partial t}=D \frac{\partial^{2} N_{\mathrm{D}}}{\partial x^{2}},
$$

where $N_{\mathrm{D}}$ is the dopant concentration, $D$ is the diffusivity of $\mathrm{P}$ into $\mathrm{Si}, t$ is the diffusion time and $x$ the diffusion depth. As shown in Fig. 2, the fitting of the doping profiles for a diffusion of $\mathrm{POCl}_{3}$ at $850^{\circ} \mathrm{C}$ and $35 \mathrm{~min}$ (corresponding to the continuous lines) are in agreement with CV measurements. In order to understand the effect of the doping profiles on the transition between the $n$ - and $p$-regions, we have calculated the depletion width in both regions by solving Poisson's equation at the junction. Assuming the continuity of the electric field $\xi$ at the junction and fixing the differential potential between the $n$ and $p$ regions to be equal to the built-in voltage $\left(V_{\mathrm{bi}}\right)$ yields [15]

$$
\begin{aligned}
\frac{1}{K_{\mathrm{s}} \varepsilon_{0}} \int_{x_{j}}^{x_{n}} \rho(x) \mathrm{d} x & =\frac{1}{K_{\mathrm{s}} \varepsilon_{0}} \int_{-x_{p}}^{x_{\mathrm{j}}} \rho(x) \mathrm{d} x \\
V_{\mathrm{bi}} & =-\int_{-x_{p}}^{x_{n}} \xi(x) \mathrm{d} x,
\end{aligned}
$$

where $K_{\mathrm{s}}$ is the relative dielectric constant, $\varepsilon_{0}=8.854 \times 10^{-14}(\mathrm{~F} / \mathrm{cm}), \rho$ the net charge density, $x_{\mathrm{j}}$ the junction depth, and $x_{n}$ and $x_{p}$ denote the depletion limits. The depletion widths resulting from these calculations are reported in Table 1 and indicated by gray areas in Fig. 2. The plot shows the microwire cross-section with the doping
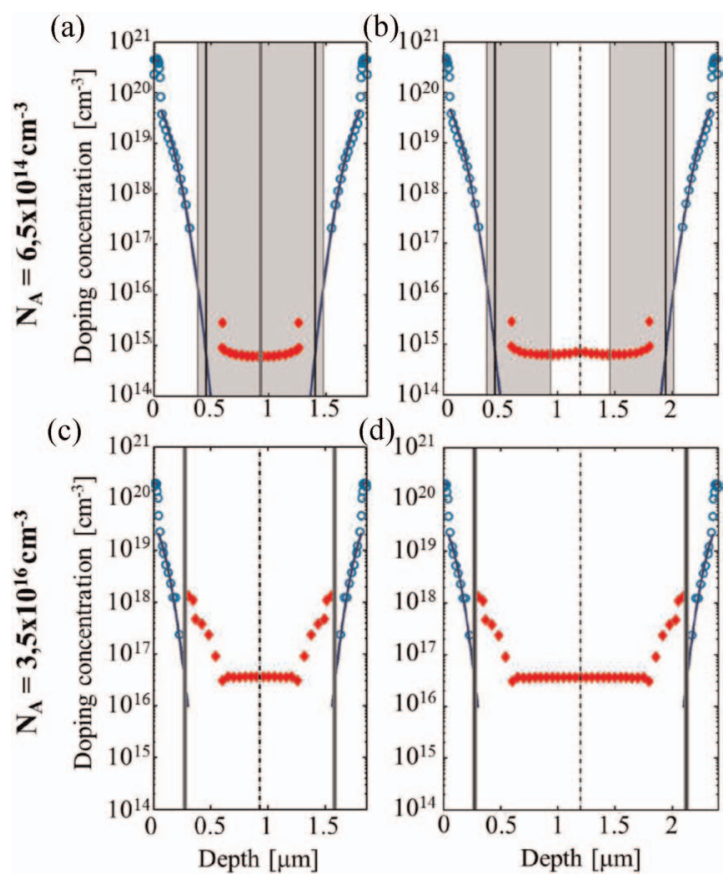

Figure 2 (online color at: www.pss-a.com) Doping profiles across the wire cross-section. The symbols represent experimental measurements and the continuous lines show the simulated diffusion profiles. Vertical black lines define the position of the junction, while gray areas represent depleted regions for four different situations: (a) $N_{\mathrm{A}}=6.5 \times 10^{14} \mathrm{~cm}^{-3}, \phi=1.86 \mu \mathrm{m}$; (b) $N_{\mathrm{A}}=6.5 \times 10^{14} \mathrm{~cm}^{-3}$, $\phi=2.4 \mu \mathrm{m} ;$ (c) $N_{\mathrm{A}}=3.5 \times 10^{16} \mathrm{~cm}^{-3}, \phi=1.86 \mu \mathrm{m}$; (d) $N_{\mathrm{A}}=3.5 \times$ $10^{16} \mathrm{~cm}^{-3}, \phi=2.4 \mu \mathrm{m}$. 
Table 1 Average measured properties of solar cell devices.

\begin{tabular}{lllllll}
\hline $\begin{array}{l}\phi \\
(\mu \mathrm{m})\end{array}$ & $\begin{array}{l}N_{\mathrm{A}} \\
\left(\mathrm{cm}^{-3}\right)\end{array}$ & $\begin{array}{l}\text { depletion } \\
\text { width } \\
(\mathrm{nm})\end{array}$ & $\begin{array}{l}J_{\mathrm{sc}} \\
\left(\mathrm{mA} / \mathrm{cm}^{2}\right)\end{array}$ & $\begin{array}{l}V_{\mathrm{oc}} \\
(\mathrm{V})\end{array}$ & FF & $\begin{array}{l}\eta \\
(\%)\end{array}$ \\
\hline \multirow{2}{*}{1.86} & $4.2 \times 10^{14}$ & 758 & 20.0 & 0.53 & 0.74 & 7.9 \\
& $6.5 \times 10^{14}$ & 555 & 19.8 & 0.55 & 0.71 & 7.7 \\
& $3.5 \times 10^{16}$ & 77 & 22.3 & 0.54 & 0.58 & 6.9 \\
\multirow{4}{*}{3.40} & $4.2 \times 10^{14}$ & 758 & 21.6 & 0.53 & 0.73 & 8.4 \\
& $6.5 \times 10^{14}$ & 555 & 23.3 & 0.53 & 0.64 & 7.9 \\
& $3.5 \times 10^{16}$ & 77 & 22.4 & 0.54 & 0.57 & 6.8 \\
& $4.2 \times 10^{14}$ & 758 & 24.9 & 0.53 & 0.74 & 9.7 \\
& $6.5 \times 10^{14}$ & 555 & 24.4 & 0.54 & 0.68 & 9.0 \\
planar & $3.5 \times 10^{16}$ & 77 & 22.7 & 0.50 & 0.50 & 5.8 \\
& $4.2 \times 10^{14}$ & 758 & 18.9 & 0.53 & 0.76 & 7.6 \\
& $6.5 \times 10^{14}$ & 555 & 20.3 & 0.55 & 0.74 & 8.3 \\
& $3.5 \times 10^{16}$ & 77 & 17.9 & 0.55 & 0.62 & 6.1 \\
\hline
\end{tabular}

profiles and the depletion region widths for two different $p$-doping concentrations $\left(6.5 \times 10^{14}\right.$ and $\left.3.5 \times 10^{16} \mathrm{~cm}^{-3}\right)$ and two wire diameters (1.86 and $2.4 \mu \mathrm{m})$. Comparing these two cases, we observe that wires with higher $p$-doping concentration present thinner depletion widths (77 nm compared to $555 \mathrm{~nm}$ ) and shallow junction depths $(270 \mathrm{~nm}$ instead of $455 \mathrm{~nm})$. As a consequence, for the smallest diameter nanowires $(1.86 \mu \mathrm{m})$ the core is fully depleted. This effect occurs for diameters of 1.86 and $2.4 \mu \mathrm{m}$ when the $p$-doping concentration is $4.2 \times 10^{14} \mathrm{~cm}^{-3}$ and for a diameter of $1.86 \mu \mathrm{m}$ in the case of a $p$-doping concentration of $6.5 \times 10^{14} \mathrm{~cm}^{-3}$.

We study the effect of the depletion width and wire diameter on the performance of the device. EQEs for all the $p n$ junction designs are compared in Fig. 3. As shown in plots (a) and (b) of Fig. 3, for small diameters and low p-doping concentrations EQE decreases dramatically, especially in the visible regime as the light is mainly absorbed in the wires. For long wavelengths, where photons are absorbed beneath the wires [16], the EQE for all the diameters becomes similar. The solar power conversion efficiencies were also measured under an AM 1.5G illumination, obtaining a maximum mean efficiency of $9.7 \%$. All the results summarized in Table 1 were averaged over four samples fabricated under identical conditions. They show that fill factor (FF) and efficiency $(\eta)$ both increase with decreasing the $p$-doping concentration.

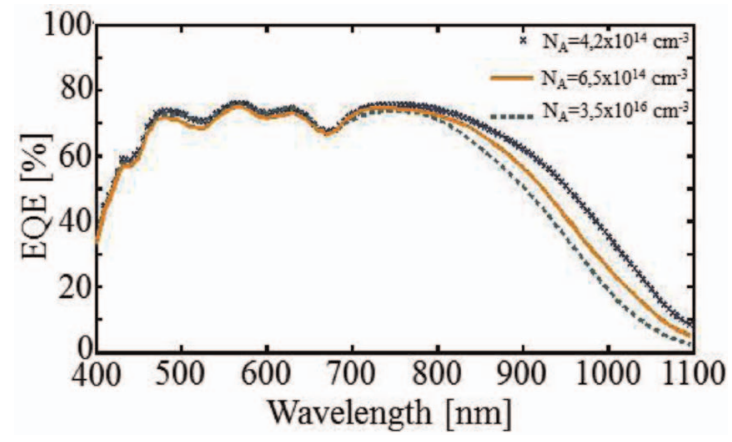

Figure 4 (online color at: www.pss-a.com) Comparison of the $\mathrm{EQE}$ of microwire arrays for the three different $p$-doping concentrations: $4.2 \times 10^{14}, 6.5 \times 10^{14}$, and $3.5 \times 10^{16} \mathrm{~cm}^{-3}$. The diameter of the wires is $3.1 \mu \mathrm{m}$ in the three cases.

This improvement is mainly due to a decrease of the recombination at the bulk [17]. To further illustrate this, we compare the EQE curves of arrays with a wire diameter of $3.1 \mu \mathrm{m}$ and different $p$-doping (Fig. 4). Clearly, long wavelength photons, which are absorbed in the bulk, are collected more efficiently in the case of low doping concentrations of the substrate. These measurements also indicate that the larger is the not depleted core, the higher is the short-circuit current density, presumably as a result of increased carrier collection. It should be noted that this statement can only be true as long as the distance from the center of the nanowire to the depletion region is smaller than the diffusion length of the minority carriers.

Finally, we examine the solar cell performance for different doping concentrations of the shell. For this, we keep the doping of the substrate constant to $3.5 \times 10^{16} \mathrm{~cm}^{-3}$ and vary the $n$-type doping by diffusing $\mathrm{POCl}_{3}$ at two different temperatures: 850 and $900{ }^{\circ} \mathrm{C}$. The $n$-doping profiles for these two cases are shown in Fig. 5a and b. As the mobility of dopants increases with the temperature, the $p n$ junction is formed at $270 \mathrm{~nm}$ from the surface in the case of a diffusion at $850^{\circ} \mathrm{C}$ and at $635 \mathrm{~nm}$ for a diffusion at $900{ }^{\circ} \mathrm{C}$. In the first case, the doping concentration decreases more abruptly.

To evaluate the collection efficiency of photogenerated carriers, we need to take into account the minority diffusion lengths of the carriers, which depends on the carrier
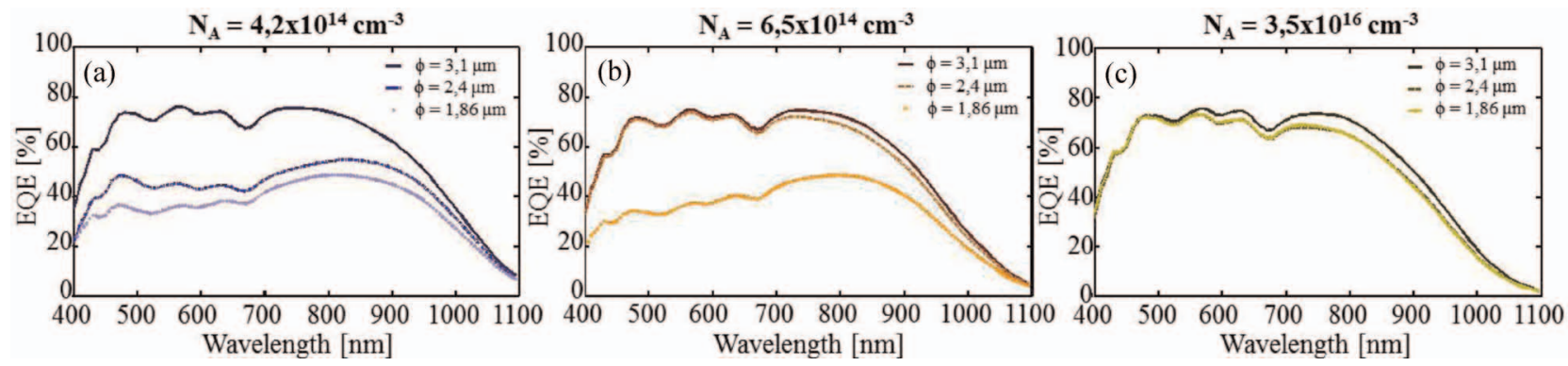

Figure 3 (online color at: www.pss-a.com) EQE as function of the diameter for (a) $N_{\mathrm{A}}=4.2 \times 10^{14} \mathrm{~cm}^{-3}$, (b) $N_{\mathrm{A}}=6.5 \times 10^{14} \mathrm{~cm}^{-3}$, and (c) $N_{\mathrm{A}}=3.5 \times 10^{16} \mathrm{~cm}^{-3}$. 

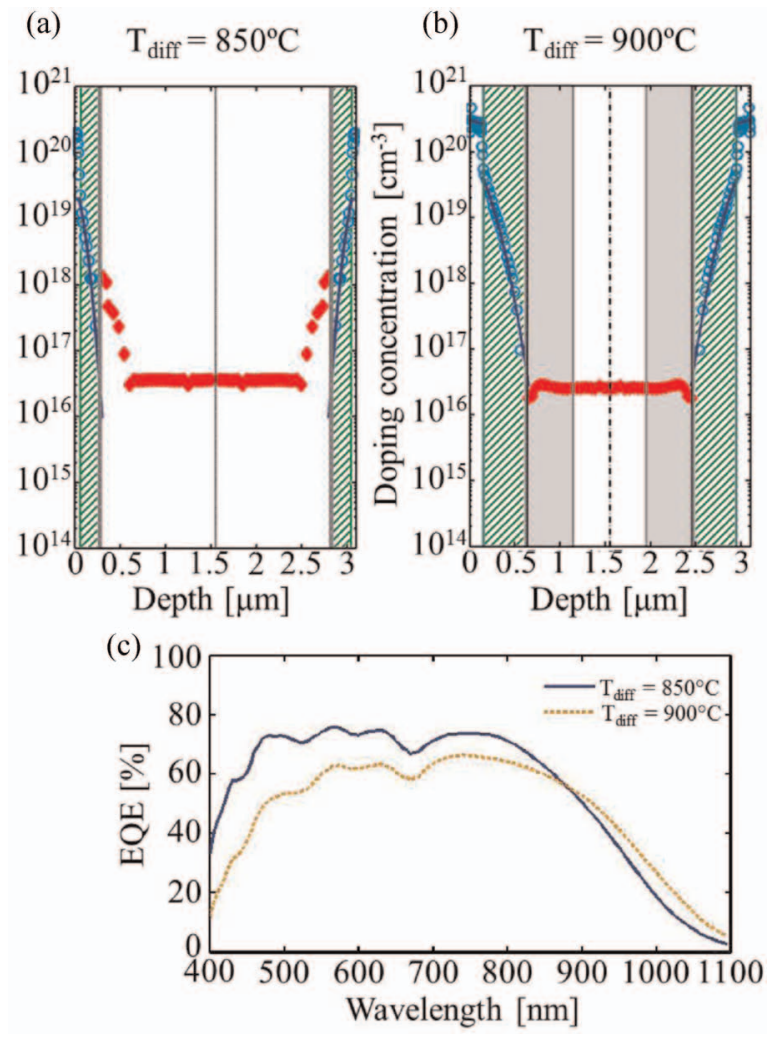

Figure 5 (online color at: www.pss-a.com) Doping profiles across the wire cross-section for a diffusion temperature of (a) $900{ }^{\circ} \mathrm{C}$ and (b) $850{ }^{\circ} \mathrm{C}$. The symbols represent experimental measurements and the continuous lines show the simulated diffusion profiles. Vertical black lines define the position of the junction, gray areas represent depleted regions and shaded green areas depict the zone where the diffusion length of minority carriers is higher than the distance to the junction. (c) Comparison of the EQE when changing the doping profile of the shell.

concentration [18]. The diffusion length of holes at an electron concentration of $2 \times 10^{20} \mathrm{~cm}^{-3}$ (doping concentration at the surface)is as low as $200 \mathrm{~nm}$, meaning that carriers generated at the surface cannot be collected as the beginning of the depletion area is $627 \mathrm{~nm}$ deep for a diffusion of $900{ }^{\circ} \mathrm{C}$. Shaded green areas in Fig. $5 \mathrm{a}$ and b correspond to the regions where the photogenerated carriers can be efficiently collected. This area is remarkably closer to the nanowire surface for the lower temperature diffusion (around $60 \mathrm{~nm}$ ) than for the higher one (around $150 \mathrm{~nm}$ ). Now we compare this with the spectral dependence of the EQE of the device (Fig. 5c). The cells obtained with an $850^{\circ} \mathrm{C}$ diffusion exhibit a higher $\mathrm{EQE}$ in a wider range of wavelengths, and especially in the visible regime. This result is in agreement with the existence of a smaller region in the nanowire over which the photogenerated carriers cannot be collected, giving rise to fewer recombination losses in the emitter region. This translates into a $4.6 \%$ higher $J_{\text {sc }}$ for cells with $n$-doped layers obtained by an $850^{\circ} \mathrm{C}$ diffusion $\left(22.73 \mathrm{~mA} / \mathrm{cm}^{2}\right)$ compared to those diffused at $900^{\circ} \mathrm{C}$ $\left(21.73 \mathrm{~mA} / \mathrm{cm}^{2}\right)$.
4 Conclusions In summary, we have demonstrated the influence of the $p n$ junction design on the carrier collection and electrical conversion efficiency. The minimum diameter of the wires is limited by the width of the depletion region resulting from the $p$ - and $n$-doping concentrations. Moreover, the junction should be as close as possible to the side facets surface.

Acknowledgements This research has been funded by the ERC grant Upcon. ADM and AFiM thank Pere Roca i Cabarrocas for helpful discussions. FME thanks G. Abstreiter for allowing the realization of the diploma thesis at EPFL. C. Ballif and H. Simon are also kindly acknowledged for experimental support.

\section{References}

[1] M. A. Green, Prog. Photovolt.: Res. Appl. 9, 123-135 (2001).

[2] B. M. Kayes, H. A. Atwater, and N. S. Lewis, J. Appl. Phys. 97(11), 114302 (2005).

[3] R. R. LaPierre, J. Appl. Phys. 109(3), 034311 (2011).

[4] M. D. Kelzenberg, D. B. Turner-Evans, D. B. Kayes, B. M. Filler, M. C. Putnam, N. S. Lewis, and H. A. Atwater, Single-nanowire Si solar cells, in: Proc. 33rd IEEE Photovoltaic Spec. Conf., San Diego, CA, 2008.

[5] D. R. Kim, C. H. Lee, P. M. Rao, I. S. Cho, and X. L. Zheng, Nano Lett. 11, 2704-2708 (2011).

[6] M. D. Kelzenberg, S. W. Boettcher, J. A. Petykiewicz, D. B. Turner-Evans, M. C. Putnam, E. L. Warren, J. M. Spurgeon, R. M. Briggs, N. S. Lewis, and H. A. Atwater, Nature Mater. 9, 239-244 (2010).

[7] M. Foldyna, L. Yu, B. O’Donnell, and P. R. i. Cabarrocas, Optical absorption in vertical silicon nanowires for solar cell applications, in: Proc. SPIE, 2011.

[8] J. Kupec, R. L. Stoop, and B. Witzigmann, Opt. Express 18(26), 27589-27605 (2010).

[9] J. Zhu, Z. Yu, G. F. Burkhard, C. M. Hsu, S. T. Connor, Y. $\mathrm{Xu}, \mathrm{Q}$. Wang, M. McGehee, S. Fan, and Y. Cui, Nano Lett. 9, 279-282 (2009).

[10] M. C. Putnam, S. W. Boettcher, M. D. Kelzenberg, D. B. Turner-Evans, J. M. Spurgeon, E. L. Warren, R. M. Briggs, N. S. Lewis, and H. A. Atwater, Energy Environ. Sci. 3, 1037-1041 (2010).

[11] M. D. Kelzenberg, D. B. Turner-Evans, M. C. Putnam, S. W. Boettcher, R. M. Briggs, J. Y. Baek, N. S. Lewis, and H. A. Atwater, Energy Environ. Sci. 4, 866-871 (2011).

[12] C. Kendrick, H. Yoon, Y. Yuwen, G. Barber, H. Shen, T. Mallouk, E. Dickey, T. Mayer, and J. Redwing, Appl. Phys. Lett. 97(14), 143108 (2010).

[13] W. C. Johnson and P. T. Panousis, IEEE Trans. ED-18, 965-973 (1971).

[14] S. W. Jones, Diffusion in Silicon (IC Knowledge LLC, Georgetown, MA, 2008).

[15] G. W. Neudeck, The pn Junction Diode (Addison-Wesley Publ. Co., Reading, MA, 1989).

[16] M. A. Green and M. J. Keevers, Prog. Photovolt.: Res. Appl. 3, 189-192 (1995).

[17] D. Macdonald and A. Cuevas, Prog. Photovolt.: Res. Appl. 8, 363-375 (2000).

[18] J. del Alamo, S. Swirhun, and R. M. Swanson, Solid-State Electron. 28, 47-54 (1985). 